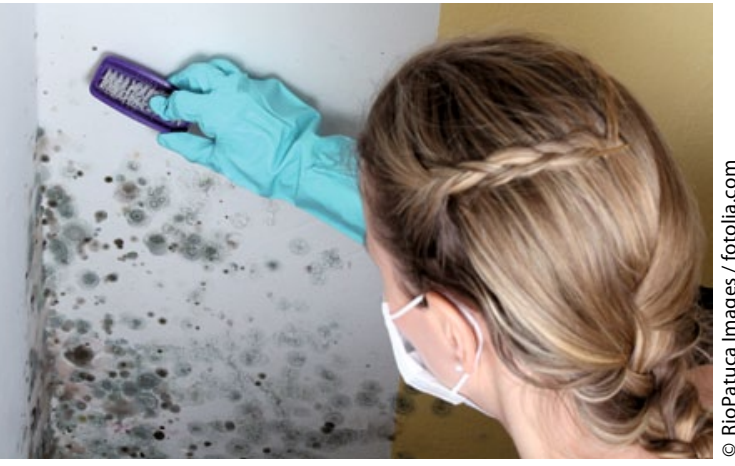

Rhinitisgefahr

\title{
Die Schnupfen-Schimmel-Connection
}

- Laut Dr. Maaritta Jaakkola und Ihren Kollegen aus Oulu, Finnland, zeigen Daten einer Metaanalyse, dass dort, wo Inspektoren erhöhte Feuchtigkeit oder Schimmel fanden, die Schnupfenrate um den Faktor 2,6 erhöht ist [Jaakkola MS et al. J Allergy Clin Immunol 2013; 132: 1099-110]. Es überrascht nicht, dass feucht-schimmlige Räume vor allem in kalten und gemäßigten Zonen mit einem erhöhten
Rhinitisrisiko einhergehen, weniger im warmen oder tropischen Klimata.

Ebenfalls analysiert wurde die Prävalenz speziell von allergischer Rhinitis in feuchtschimmliger Umgebung. Dabei ergab sich nur eine etwa um $50 \%$ erhöhte Rate, bei Schimmelgeruch war die Prävalenz um etwa $90 \%$ erhöht. Insgesamt gingen 31 Studien in die Metaanalyse ein.

Thomas Müller

\section{Roter-Hand-Brief}

\section{Rückruf von fünf Chargen des Adrenalin- Autoinjektors Jext ${ }^{\circledast}$}

— Die ALK-Abelló Arzneimittel GmbH hat in Deutschland in Abstimmung mit den zuständigen Behörden vorsorglich fünf Chargen des Adrenalin-Autoinjektors Jext ${ }^{\circledR}$ zurückgerufen. Der Adrenalin-Pen wird für die Notfallbehandlung von anaphylaktischen Reaktionen verwendet. Der Grund für den Rückruf: Für den Autoinjektor besteht das Risiko, dass die Adrenalinlösung in sehr seltenen Fällen bei der Anwendung nicht wie vorgesehen in den Muskel des
Patienten abgegeben wird. Betroffen sind vier Chargen der Stärke Jext ${ }^{\oplus} 300$ Mikrogramm und eine Charge der Stärke Jext ${ }^{\circledast}$ 150 Mikrogramm. Ebenso betroffen sind die Jext ${ }^{\oplus}$-Parallelimporte der Unternehmen Eurim Pharm und Pharma Gerke. Alle betroffenen Chargen von ALK und der Parallelimporte sind auf der Internetseite des Herstellers www.alk.de im Bereich „ALK News" abrufbar. Für Fragen steht zudem eine Telefon-Hotline von ALK zur Verfü-

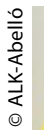

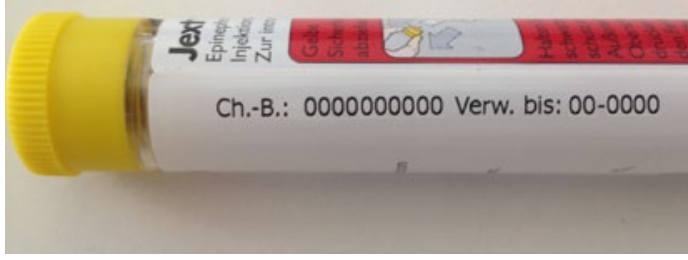

Patienten können anhand der aufgedruckten Chargenbezeichnung überprüfen, ob ihr Autoinjektor zu den betroffenen Chargen zählt.

gung, die unter der Nummer (0 40) 7038 45-0 erreichbar ist.

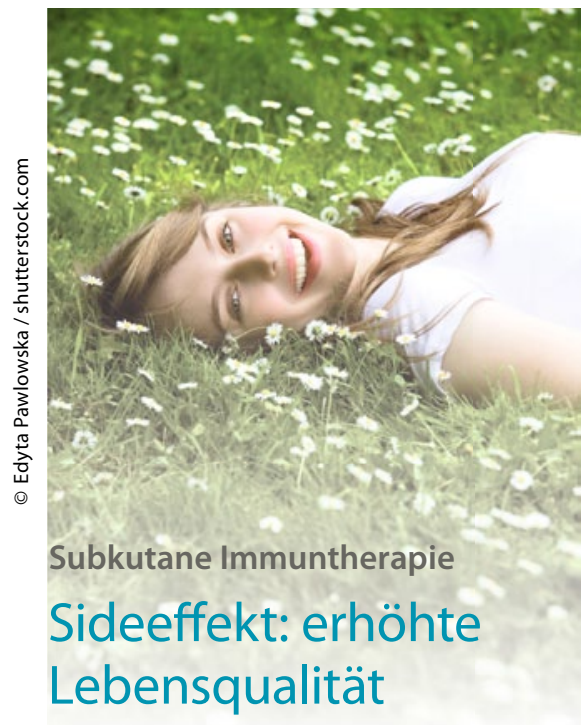

— Daten einer Veröffentlichung von Karin D. Petersen und ihren dänischen Kollegen zeigen, inwiefern eine subkutane Immuntherapie (SCIT) nach einem Jahr die Lebensqualität von Patienten mit Rhinitis oder Asthma, verursacht durch eine Allergie gegen Gräserpollen oder Hausstaubmilben, positiv beeinflusst [Petersen KD et al. World Allergy Organ J 2013; 6: 15]. Anhand von zwei Instrumenten zur Erfassung der krankheitsspezifischen Lebensqualität, dem EQ-SD-Score und dem 15D-Score, wurde jeweils ein Anstieg von 0,70 auf 0,77 bzw. von 0,83 auf 0,86 deutlich. Weiter kam es bei den Betroffenen $(n=248)$ nicht nur zu einem deutlichen Rückgang an jährlichen Krankheitstagen (im Durchschnitt von 3,7 auf 1,2), sondern auch die durchschnittlichen Tage mit Beschwerden sanken von 189 auf 145 pro Jahr. Insgesamt erschließt sich, dass die SCIT pro Patient den „quality adjusted life year" (QALY)-Wert um 0,03-0,06 erhöht.

Simone Kohler

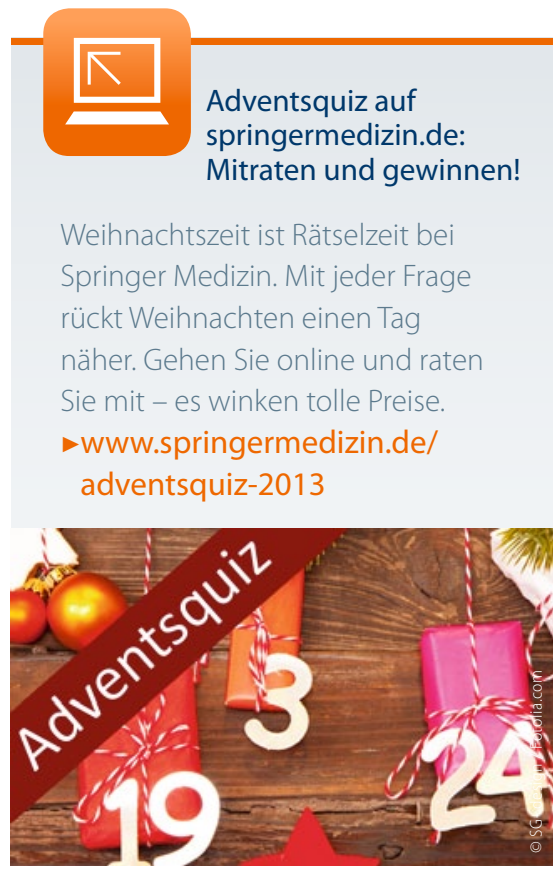

